\title{
Data-Driven Parameter Choice for Illumination Artifact Correction of Digital Images
}

\author{
Hong-Phuong Dang, Myriam Vimond and Ségolen Geffray
}

\begin{abstract}
We propose a new procedure for image illumination correction with data-driven parameter choice. This procedure aims at estimating the reflectance image from a corrupted version in which the corruption is due to pointwise multiplicative illumination artifact. The $\log$-illumination artefact consists of "smooth" variations of the intensity which are modelled by a function lying in a finite dimensional space. Then a $\gamma$-correction is incorporated. The question of model selection is difficult to solve. We propose an entropy minimization criterion for the selection of both the approximating $\log$-illumination space dimension and the $\gamma$-coefficient, so that no parameter tuning is needed. Several experiments are presented using this approach. A comparison to other methods illustrates the relevance of this approach.
\end{abstract}

Index Terms-illumination artifact, polynomial regression, semiparametric estimation, statistical image processing.

\section{INTRODUCTION}

Illumination artifacts arise in a variety of digital images such as digital photography, digital microscopy or Magnetic Resonance images. Illumination artifacts consist of "smooth" variations in color/gray level intensity which are visible in the acquired image but are not present in the original scene. These artifacts can greatly degrade the accuracy of computer vision algorithms such as segmentation or object detection algorithms. The Retinex model [1] involves decomposing an image into two components: the illumination and the reflectance, the latter being defined as the fraction of radiant light which is reflected from a surface. The problem of illumination correction can then be reformulated as the estimation of these two components from a single observed image.

Many algorithms [2-6] aim at solving this ill-posed problem. A first group of algorithms uses a path-wise approach to compare the color of the current pixel to that of a set of pixels [7, and references therein]. A second group uses filtering techniques, and includes two bilateral filtering Retinex [8], homomorphic filtering [9] or multi-scale Retinex [10] (MSR). A third group uses partial differential equations [11, 12] based on the assumption that the illumination is relatively small. A fourth group relies on variational procedures [1320] which introduce regularity priors on the reflectance and illumination parts. Another group is based on intensity distribution modeling such as the iterative method N4 [21] which estimates the illumination bias and the reflectance. Most of these procedures depend on tuning parameters the value of which are difficult to choose and may vary from one input

H-P. Dang and M. Vimond are with Ensai, Crest UMR 9194, 35172 Bruz, France. E-mail: firstname.lastnamedensai.fr.

S. Geffray is affiliated to the University of Strasbourg, IRMA UMR 7501, France. Email: geffray@math.unistra.fr. image to another. Automatic selection of model parameters is a strategic challenge when a large set of images is to be treated, and even remains an open problem for state-of-art methods.

In [22], to estimate the reflectance, the log-illumination of a Retinex model is approximated by a "smooth" function which is chosen from an appropriate finite sub-space. It has been demonstrated that the illumination correction procedure is consistent provided that the approximating log -illumination space dimension is fixed in advance. Here, we consider an extension of [22] that incorporates a Gamma-correction. The main novelty consists of selecting both the approximating $\log$-illumination space dimension and the Gamma-correction coefficient by minimizing the entropy of the estimated reflectance. The procedure is then entirely data-driven.

The paper is structured as follows. Section II describes the model and the estimation procedure, then introduces the criteria for selecting the hyperparameters. Section III illustrates the relevance of the proposed method in comparison to Retinex procedures on noiseless non-uniformly illuminated images. The robustness to the presence of noise is also displayed there. Section IV gathers conclusions and prospects.

\section{Proposed Method}

\section{A. Semiparametric Regression Model}

Let $F$ be an image defined on the domain $\mathcal{D}=(0,1)^{2}$. According to Retinex literature, the image $F$ can be multiplicatively decomposed in a pointwise manner into two components,

$$
F(x)=R(x) L(x), \quad x \in \mathcal{D},
$$

where $R$ represents the reflectance (signal of interest) which is distorted by a luminance $L$. As [23], the log-luminance $\ell=\log L$ is such that its gradient is the projection of the gradient of the log-image $f=\log F$ onto a finite dimensional subspace $\mathcal{L}$ of the space of square integrable functions on the domain $\mathcal{D}=(0,1)^{2}$, that is

$$
\ell \in \underset{p \in \mathcal{L}}{\arg \min } \int_{\mathcal{D}}|\nabla f-\nabla p|_{2}^{2},
$$

where $|\cdot|_{2}$ is the euclidean norm in $\mathbb{R}^{2}$. Specifically, the vector space $\mathcal{L}$ of dimension $(M+1)$ is composed of smooth "low-frequency" functions such that we can extract a basis $\Psi=\left\{\psi_{m}: m=0, \ldots, M\right\}$ where $\psi_{0} \equiv 1$ and where the functions $\psi_{m}$ and their gradients $\nabla \psi_{m}$ are continuous functions on the closure $\overline{\mathcal{D}}$ of $\mathcal{D}$ for any $m=1, \ldots, M$. This implies that $\ell$ consists of spatially smooth variations which are not present in the reflectance $R$. 


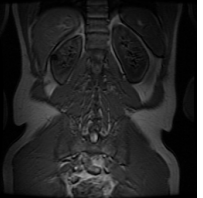

input data

(a)

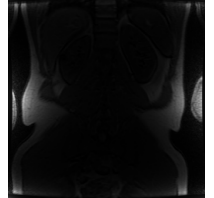

$\eta=7.4129$

(b)

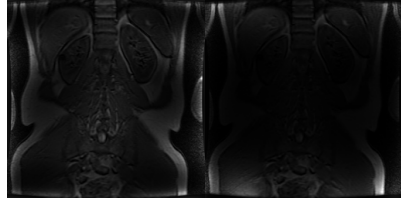

$\eta=6.3942 \quad \eta=7.3207$

(c)

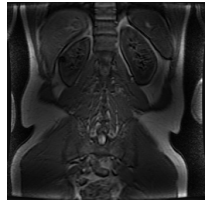

(e) $\eta=6.2825$

(e)

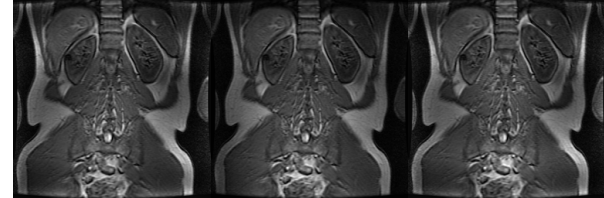

$\eta=6.2315$

(f) $\eta=6.2354$

(g) $\eta=6.1963$

(h)

Fig. 1: (a) is a Magnetic Resonance Image (MRIX, DICOM image sample sets). The other images are the estimated reflectance from the input image with $\eta$ the associated entropy. For pictures (b), (c) and (d), $\ell$ is modeled as a polynomial function of degree $\left(d_{1}, d_{2}\right)$ : (b) - $(3,4)$, (c) - $(5,6),(\mathrm{d})-(8,2)$ and no Gamma-Correction is done, i.e. $\gamma=0.0$. For pictures (e), (f) and $(\mathrm{g}), \ell$ is modeled as a polynomial function of degree $(5,6)$ and the Gamma-Correction is done with the choice $\gamma$ : (e) - 0.2 , (f) $-0.5,(\mathrm{~g})-0.8$. For picture $(\mathrm{h})$, the model selection through entropy minimization yields $\left(\hat{d}_{1}, \hat{d}_{2}\right)=(4,7)$ and $\hat{\gamma}=0.5$.

We set the identifiability condition presented in [22]:

$$
\int_{\mathcal{D}} L(x) d x=1
$$

The points for which the illumination $L$ is greater than 1 correspond to an over-illuminated area, while the points for which the illumination is less than 1 correspond to a darkened area. When there is no illumination artefact, the function $L$ is identically equal to 1 .

\section{B. Estimation procedure}

Consider that the data are a possibly noisy version of $\{F(x), x \in \mathcal{D}\}$ with an independent and identically distributed zero-mean additive noise. The problem of getting rid of illumination artefacts can be reformulated as the estimation of $\{R(x), x \in \mathcal{D}\}$ from the data without knowing the luminance $L(x)$. In this context, [22] proposes a reflectance estimator (briefly summarized below) which is used here as a preliminary reflectance estimator, see [22] for more details.

a) Nonparametric estimation of $\nabla f$ : The first step consists of estimating both $F$ and $\nabla F$ using a nonparametric procedure. Let us denote by $\widehat{F}$ and $\widehat{\nabla F}$ such an estimate of $F$ and $\nabla F$ respectively. Since $\nabla f=(\nabla F) / F$, we set:

$$
\widehat{\nabla f}(x)=\frac{\widehat{\nabla F}(x)}{\widehat{F}(x)}, \quad x \in \mathcal{D} .
$$

b) Parametric estimation: The second step consists of estimating the $\log$-illumination $\ell$ using a least squares criterion. Since $\ell \in \mathcal{L}$, we write $\ell$ as a linear combination of $\Psi$,

$$
\ell=\sum_{m=0}^{M} \lambda_{m} \psi_{m}
$$

Since $\nabla \psi_{0} \equiv 0$, the parameter $\lambda=\left(\lambda_{m}\right)_{m=1, \ldots, M}$ is estimated by minimizing the least squares criterion:

$$
\lambda \rightarrow \int_{\mathcal{D}}\left|\widehat{\nabla f}(x)-\sum_{m=1}^{M} \lambda_{m} \nabla \psi_{m}(x)\right|^{2} d x .
$$

In view of the identifiability constraint (3), the illumination is estimated as

$$
\widehat{L}(x)=\exp (\hat{\ell}(x))=\exp \left(\widehat{\lambda}_{0}+\sum_{m=1}^{M} \hat{\lambda}_{m} \psi_{m}(x)\right)
$$

where $\exp \left(-\widehat{\lambda}_{0}\right)=\int_{\mathcal{D}} \exp \left(\sum_{m=1}^{M} \hat{\lambda}_{m} \psi_{m}(x)\right) d x$. c) Preliminary reflectance estimator: We introduce our uncorrected reflectance estimator as:

$$
\widehat{R}(x)=\frac{\widehat{F}(x)}{\widehat{L}(x)}, \quad x \in \mathcal{D} .
$$

d) Gamma-Correction: Rather than doing the Gammacorrection as a pre-processing step on the input data [24], we do as [25] and carry out the Gamma-correction with power parameter $\gamma \in[0,1)$ on the estimated luminance and multiply our preliminary reflectance estimator in a pointwise manner by the Gamma-corrected estimated luminance. Our Gammacorrected reflectance estimator is finally given by:

$$
\widehat{R}(x)=\frac{\widehat{F}(x)}{\widehat{L}(x)^{1-\gamma}} .
$$

\section{Proposed criteria for model selection}

Prior to the model selection step, a critical issue that remains to be addressed is the choice of the function basis $\Psi$. It is widely held that a specific set of functions, such as either the trigonometric basis, or a spline basis or a wavelet basis, should be considered depending on the problem at hand. A practical disadvantage of the wavelet basis is that the basis cardinal increases exponentially with the scale parameter. With our test images, this would limit the scale exploration up to order 3, which is very restrictive. On the other hand, the polynomial basis provides satisfying results in our test images and its dimension increases linearly with the polynomial degree. As a consequence, in this paper, we focus our exploration on the polynomial basis, that is

$$
\psi_{m}(x)=x_{1}^{m_{1}} x_{2}^{m_{2}}, \quad m=m_{1}+\left(d_{1}+1\right) m_{2},
$$

where $x=\left(x_{1}, x_{2}\right) \in \mathcal{D}, m_{1}=0, \ldots d_{1}, m_{2}=0, \ldots, d_{2}$, and $d=\left(d_{1}, d_{2}\right)$ is the degree of polynomial approximation of the log-illumination.

a) Polynomial Approximation of the log-illumination:

Fig.1 (b), (c) and (d) show the respective estimations of reflectance associated to three different choices of polynomial degree. Comparing Fig.1 (c) to Fig.1 (b) and (d) stresses the importance of finding an appropriate polynomial degree to remove the illumination artefact. The choice of the polynomial degree clearly influences the final result. 

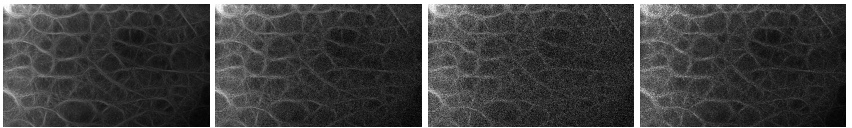

(a) original (b) $20.44 \mathrm{~dB}$ (c) $15.24 \mathrm{~dB}$
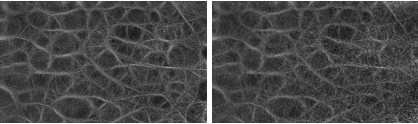

(e)

(f)

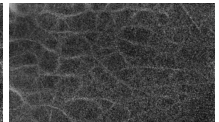

(d) $19.25 \mathrm{~dB}$

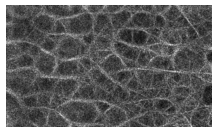

(g)

(h)

Fig. 2: The first row presents the input images and their PSNR: (a) image without noise, (b) and (c) with a i.i.d. Gaussian noise and (d) with speckle noise. The second row presents the corresponding estimated reflectances: (e) $\widehat{R}_{\hat{d}=(6,5), \hat{\gamma}=0}$, (f) $\widehat{R}_{\hat{d}=(6,5), \hat{\gamma}=0}$, (g) $\widehat{R}_{\hat{d}=(7,1), \hat{\gamma}=0.2}$, (h) $\widehat{R}_{\hat{d}=(6,5), \hat{\gamma}=0}$.

b) Gamma correction: Fig.1 (e), (f) and (g) display estimated reflectances obtained with the same polynomial degree, that is $(5,6)$, but with different choices for the value of $\gamma$. Fig. 1 (f) gives a better visual result, with $\gamma=0.5$. This experiment shows that the proposed Gamma-correction with an optimal $\gamma$ value improves the results and avoids image background segmentation as a pre-processing step, unlike [22].

c) Model Selection using Entropy: We use Shannon's entropy as an objective criterion to choose both the polynomial degree $d=\left(d_{1}, d_{2}\right)$ and the Gamma-correction coefficient $\gamma$ in a data-driven way. In the Retinex literature, information minimization methods are based on the statement that an illumination bias increases the entropy of the original image, [see 5, and reference therein]. As a consequence, we expect that the entropy of the estimated reflectance is minimal for optimal $(d, \gamma)$ that is such that the image is properly corrected. We select $(d, \gamma)$ using entropy minimization:

$$
(\hat{d}, \hat{\gamma})=\arg \min _{(d, \gamma)} \eta\left(\widehat{R}_{d, \gamma}\right)
$$

where $\eta(\mathcal{I})$ is the Shannon's entropy of an image $I$, and where we write $\widehat{R}_{d, \gamma}$ instead of $\widehat{R}$ estimated with $(d, \gamma)$, and $\widehat{L}_{d, \gamma}$ as the associated luminance to highlight the dependance of the estimated reflectance on $(d, \gamma)$. In practice, the minimization is done over a grid of $(d, \gamma)$ values. In our experiments, we explore $\gamma \in\{0,0.2,0.5,0.8\}$ and $d \in\{1, \ldots, 9\}^{2}$.

The first experiments of Fig. 1 with different choices of the polynomial degree yield the minimum entropy value with the better visual result when $\left(d_{1}, d_{2}\right)=(5,6)$. Using the same polynomial degree $(5,6)$, the second experiments of Fig.1 with different choices of Gamma-Correction coefficient yield the minimum entropy value with the better visual result when $\gamma=0.5$. Fig. 1 (h) shows the result of the proposed method with entropy minimization as model selection criterion, that yields $\left(\hat{d}_{1}, \hat{d}_{2}\right)=(4,7)$ and $\hat{\gamma}=0.5$.

\section{EXPERIMENTAL COMPARISONS}

Noiseless images: The performance of the proposed method is compared to recent methods of the literature: the N4 method [21], MSR [10], JIEP [18], LIME [17], STAR [19], PIE [15] and SRIE[16]. Some of these algorithms are originally

\begin{tabular}{rllllllll}
\hline & Ours & STAR & JIEP & LIME & MSR & N4 & PIE & SRIE \\
\hline SSIM & 0.84 & 0.33 & 0.37 & 0.46 & 0.57 & 0.93 & 0.98 & 0.59 \\
NIQE & 3.32 & 8.85 & 4.07 & 3.92 & 3.23 & 3.32 & 3.54 & 3.66 \\
GSMD & 0.05 & 0.25 & 0.23 & 0.17 & 0.12 & 0.03 & 0.00 & 0.10 \\
\hline
\end{tabular}

TABLE I: Average of the SSIM, NIQE and GMSD indexes over a sample of 50 images, for more details see https://hphuongdang.github.io/projects/2_project/.

implemented for color images, nevertheless their implementation is also suited to gray-level images. The procedures are tested over 50 image collected from NASA image dataset ${ }^{1}$ and [15-18, 22, 26-30]. All the algorithms are used with default parameter values of their implementations.

Figure 3 illustrates that STAR and LIME enlighten the reflectance and it seems that a residual illumination remains. At the opposite, SRIE provides in general darker outputs except for the mri image. JIEP completes the job, even if the reflectance images are lighter. MSR outputs go to the gray. Moreover a border effect may be observed due to the gaussian kernel convolution. N4 and our procedure provide generally similar results. Both are sensitive to the irregularity of the images. This is illustrated by the presence of an halo in the skyline of the camels outputs. We notice N4 doesn't succeed to remove illumination in few cases, for example see the N4 outputs of girl and room images which are nearly identical to their input images. The outputs of PIE are almost identical to the input images. This might be explained by the default parameters of the algorithm which are calibrated for the $V$ channel within the Hue-Saturation-Value color space.

The methods are also compared using objective measurements to evaluate their performances quantitatively. Table I shows the average over 50 images of the Structural Similarity index (SSIM) [31], the NIQE (Natural Image Quality Evaluator) index [32], and the Gradient Magnitude Similarity Deviation index (GMSD) [33].

When the full reference SSIM index is close to 1 , this indicates a local similarity in mean and variance of input and output intensities. Given the brightness of the outputs of STAR, JIEP, LIME, MSR or the darkness of SRIE in general, it is not surprising that the average SSIM index is low for these methods. The PIE outputs being quite identical to the input images, the associated average SSIM is logically closed to 1. N4 and our method provide average SSIM index closer to 1 than any other methods, except the inoperative PIE. It is sensible to observe SSIM values less than 1 since the images are actually modified for illumination correction.

The No-Reference NIQE index compares the image at hand to a default model computed from images of natural scenes. A smaller score indicates better perceptual quality.

GMSD index measures the distortion of the gradient magnitude between the original image and the output. PIE that doesn't change much the input image logically has a 0.00 GMSD. On the other hand, even if an illumination bias is actually corrected by our method, and N4 in most cases, the GMSD is nearly 0, illustrating that the application of either

${ }^{1}$ https://dragon.larc.nasa.gov/retinex/pao/news/ 


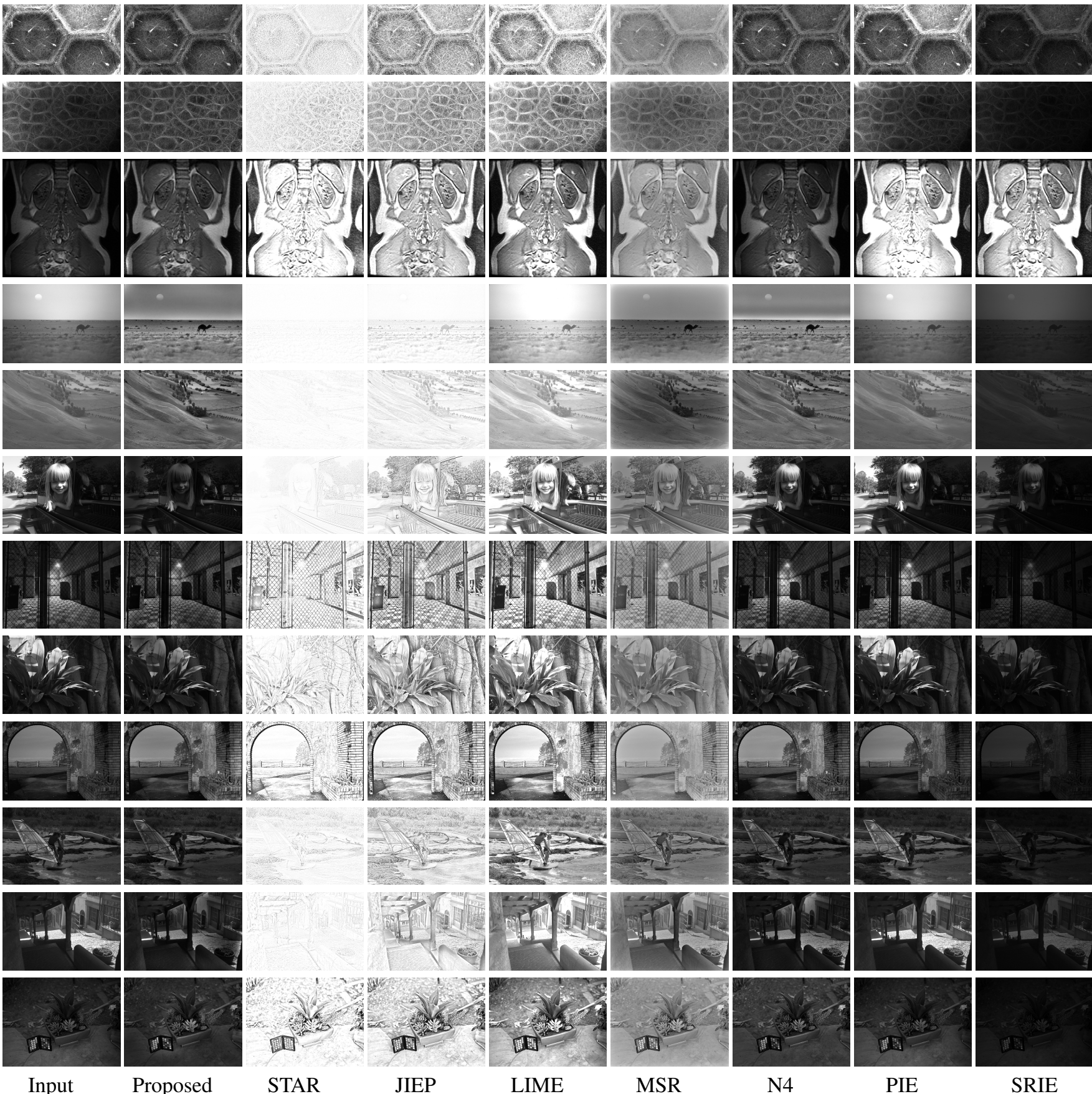

Fig. 3: Comparison of different reflectance recovery methods on a sample of images named from top to bottom as sem1, sem2, mri, camels, landscape ${ }^{\mathrm{a}}$, girl, room, plant, outdoor, sailboat, street, test.

${ }^{a}$ Faded version of Hawkes Bay NZ.jpg, originally by Phillip Capper, modified by User:Konstable.

our method or N4 does not alter the image content. The other methods suffer from gradient distortion.

Noisy images: The results of Fig. 2 illustrate that our procedure is robust to noise. A key point of our method is to inject in (4) an estimate of $\widehat{\nabla F}$ which is robust to noise. We use here the local polynomial estimate, which is a suboptimal denoising method. Interestingly, even if the treated image is still noisy, its illumination is effectively made uniform.

\section{CONCLUSION}

The proposed procedure gets rid of illumination bias on our test images in an effective manner. It is entirely datadriven and robust to noise. Interestingly, thanks the the $\gamma$ correction step, black background images no longer require background segmentation as a pre-treatment, contrarily to [22]. In a reproducible research effort, the $\mathrm{R}$ and Python codes are made available. 


\section{REFERENCES}

[1] E. Land and J. McCann, "Lightness and the retinex theory," $J$. Opt. Soc. Am., vol. 61, pp. 1-11, 1971.

[2] Z. Hou, "A review on MR image intensity inhomogeneity correction," Int. J. Biomedical Imaging, 2006.

[3] U. Vovk, F. Pernus, and B. Likar, "A review of methods for correction of intensity inhomogeneity in MRI," IEEE Transactions on Medical Imaging, vol. 26, no. 3, pp. 405-421, 2007.

[4] D. Zosso, G. Tran, and S. J. Osher, "Non-local retinex-a unifying framework and beyond," SIAM Journal on Imaging Sciences, vol. 8, no. 2, pp. 787-826, 2015.

[5] S. Song, Y. Zheng, and Y. He, "A review of methods for bias correction in medical images," Biomedical Engineering Review, vol. 1, no. 1, 2017.

[6] W. Wang, X. Wu, X. Yuan, and Z. Gao, "An experiment-based review of low-light image enhancement methods," IEEE Access, vol. 8, pp. 87 884-87917, 2020.

[7] A. Rizzi, "Designator retinex, milano retinex and the locality issue," Electronic Imaging, vol. 2016, no. 6, pp. 1-5, 2016.

[8] M. Elad, "Retinex by two bilateral filters," in Scale Space and PDE Methods in Computer Vision. Springer, 2005, pp. 217229.

[9] R. C. Gonzales and R. E. Woods, Digital image processing. Prentice-Hall, 2002.

[10] A. B. Petro, C. Sbert, and J.-M. Morel, "Multiscale retinex," Image Processing On Line, pp. 71-88, 2014.

[11] B. K. Horn, "Determining lightness from an image," Computer graphics and image processing, vol. 3, no. 4, pp. 277-299, 1974.

[12] J. M. Morel, A. B. Petro, and C. Sbert, "A PDE formalization of retinex theory," IEEE Trans. Image Processing, vol. 19, no. 11, pp. 2825-2837, 2010 .

[13] R. Kimmel, M. Elad, D. Shaked, R. Keshet, and I. Sobel, "A variational framework for retinex," International Journal of computer vision, vol. 52, no. 1, pp. 7-23, 2003.

[14] M. K. Ng and W. Wang, "A total variation model for retinex," SIAM Journal on Imaging Sciences, vol. 4, no. 1, pp. 345-365, 2011.

[15] X. Fu, Y. Liao, D. Zeng, Y. Huang, X.-P. Zhang, and X. Ding, "A probabilistic method for image enhancement with simultaneous illumination and reflectance estimation," IEEE Transactions on Image Processing, vol. 24, no. 12, pp. 4965-4977, 2015.

[16] X. Fu, D. Zeng, Y. Huang, X.-P. Zhang, and X. Ding, "A weighted variational model for simultaneous reflectance and illumination estimation," in Proceedings of the IEEE Conference on Computer Vision and Pattern Recognition, 2016, pp. 27822790.

[17] X. Guo, Y. Li, and H. Ling, "Lime: Low-light image enhancement via illumination map estimation," IEEE Transactions on image processing, vol. 26, no. 2, pp. 982-993, 2016.

[18] B. Cai, X. Xu, K. Guo, K. Jia, B. Hu, and D. Tao, "A joint intrinsic-extrinsic prior model for retinex," in Proceedings of the IEEE International Conference on Computer Vision, 2017, pp. 4000-4009.

[19] J. Xu, Y. Hou, D. Ren, L. Liu, F. Zhu, M. Yu, H. Wang, and L. Shao, "Star: A structure and texture aware retinex model," IEEE Transactions on Image Processing, vol. 29, pp. 50225037, 2020.

[20] M. Tang, F. Xie, R. Zhang, Z. Jiang, and A. C. Bovik, "A local flatness based variational approach to retinex," IEEE Transactions on Image Processing, vol. 29, pp. 7217-7232, 2020.

[21] N. J. Tustison, B. B. Avants, P. A. Cook, Y. Zheng, A. Egan, P. A. Yushkevich, and J. C. Gee, "N4itk: improved n3 bias correction," IEEE Transactions on Medical Imaging, vol. 29, no. 6, pp. 1310-1320, 2010.

[22] S. Geffray, N. Klutchnikoff, and M. Vimond, "Illumination problems in digital images. a statistical point of view," Journal of Multivariate Analysis, vol. 150, pp. 191-213, 2016.

[23] T. Tasdizen, E. Jurrus, and R. Whitaker, "Non-uniform illumina- tion correction in transmission electron microscopy," in MICCAI Workshop on Microscopic Image Analysis with Applications in Biology, 2008.

[24] R. Kimmel, M. Elad, D. Shaked, R. Keshet, and I. Sobel, "A variational framework for retinex," Int. J. Computer Vision, vol. 52, pp. 7-23, 2003.

[25] M. Ng and W. Wang, "A total variational model for retinex," SIAM J. Imaging Sci., vol. 4, pp. 345-365, 2011.

[26] D. Martin, C. Fowlkes, D. Tal, and J. Malik, "A database of human segmented natural images and its application to evaluating segmentation algorithms and measuring ecological statistics," in Proc. 8th Int'l Conf. Computer Vision, vol. 2, July 2001, pp. 416-423.

[27] S. W. Hasinoff, D. Sharlet, R. Geiss, A. Adams, J. T. Barron, F. Kainz, J. Chen, and M. Levoy, "Burst photography for high dynamic range and low-light imaging on mobile cameras," $A C M$ Transactions on Graphics (Proc. SIGGRAPH Asia), vol. 35, no. $6,2016$.

[28] S. Wang, J. Zheng, H.-M. Hu, and B. Li, "Naturalness preserved enhancement algorithm for non-uniform illumination images," IEEE Transactions on Image Processing, vol. 22, no. 9, pp. 3538-3548, 2013.

[29] X. Fu, D. Zeng, Y. Huang, Y. Liao, X. Ding, and J. Paisley, "A fusion-based enhancing method for weakly illuminated images," Signal Processing, vol. 129, pp. 82-96, 2016.

[30] P. Sen, N. K. Kalantari, M. Yaesoubi, S. Darabi, D. B. Goldman, and E. Shechtman, "Robust patch-based HDR reconstruction of dynamic scenes," ACM Transactions on Graphics (Proceedings of SIGGRAPH Asia 2012), vol. 31, no. 6, pp. 203:1-203:11, Nov. 2012.

[31] Z. Wang, A. C. Bovik, H. R. Sheikh, and E. P. Simoncelli, "Image quality assessment: from error visibility to structural similarity," IEEE transactions on image processing, vol. 13, no. 4, pp. 600-612, 2004.

[32] A. Mittal, R. Soundararajan, and A. C. Bovik, "Making a "completely blind" image quality analyzer," IEEE Signal Processing Letters, vol. 20, pp. 209-212, 2013.

[33] W. Xue, L. Zhang, X. Mou, and A. C. Bovik, "Gradient magnitude similarity deviation: A highly efficient perceptual image quality index," IEEE Transactions on Image Processing, vol. 23, no. 2, pp. 684-695, 2013. 